\title{
Thirty-Day Outcomes of Bariatric Surgery in Adolescents: a First Look at the MBSAQIP Database
}

\author{
Maher El Chaar ${ }^{1,2} \cdot$ Keith King $^{1,3}$ (D) $\cdot$ Amin Al-Mardini ${ }^{1} \cdot$ Alvaro Galvez $^{1,3} \cdot$ Leonardo Claros $^{1,2} \cdot$ Jill Stoltzfus ${ }^{1,2}$
}

Received: 31 March 2020 / Revised: 12 July 2020 / Accepted: 15 July 2020 / Published online: 25 July 2020

(C) Springer Science+Business Media, LLC, part of Springer Nature 2020

\begin{abstract}
Introduction Bariatric surgery is the only effective treatment of severe obesity. The number of adolescents undergoing bariatric surgery is increasing. However, bariatric surgery in adolescents is controversial.

Objective The purpose of this study is to evaluate the outcomes of bariatric surgery in adolescents based on the MBSAQIP database (Metabolic and Bariatric Surgery Accreditation and Quality Improvement Project).

Methods We analyzed the 2015-2017 MBSAQIP database; patients $\leq 19$ years of age were included in our analysis. Primary outcomes were 30-day serious adverse events (SAEs), organ space infection (OSI), re-intervention, and re-operation rates. Secondary outcomes included operation length, hospital stay, and re-admission rates. We conducted separate Mann-Whitney rank sums tests, chi-square, or Fisher's exact tests as appropriate, with $p<.05$ denoting statistical significance.

Results A total of 1983 adolescent patients were included in our analysis. The average age and BMI were 18.1 and 47.5, respectively. Of adolescent patients, $21.7 \%$ underwent laparoscopic Roux-en-Y gastric bypass (LRYGB) and 78.3\% underwent laparoscopic sleeve gastrectomy (LSG). The 30-day SAE and readmission rates were significantly lower for LSG compared with LRYGB (2.9\% and $2.6 \%$ vs $6.5 \%$ and $5.6 \%$, respectively; $p<0.05)$. The 30-day reoperation rate was also lower for LSG compared with LRYGB albeit not significant $(1.1 \%$ and vs $2.3 \% ; p=0.05)$. The 30-day intervention rate for LSG was significantly lower, however, compared with LRYGB $(1.2 \%$ vs $3 \% ; p<0.05)$. Compared with adult patients, $>19$ years old $(n=$ 353,726), we found no difference in our outcomes. However, adolescents had significantly shorter operation length.

Conclusion In adolescents, LSG had fewer SAE, re-intervention, and readmission rates compared with LRYGB. There was no difference in outcomes between adolescents and adults.
\end{abstract}

Keywords Adolescents $\cdot$ Bariatric surgery $\cdot$ Adults

\section{Introduction}

Excess weight in adolescents has been designated as "one of the most serious public health challenges of the 21 st century" by the World Health Organization [1]. In the USA, 7.5\% of adolescents aged $12-15$ and $9.5 \%$ of adolescents aged 16-19 have severe obesity with an estimated body mass index

Keith King

Keith.King@sluhn.org

1 St. Luke's University Health Network (SLUHN), Bethlehem, PA, USA

2 Temple Lewis Katz School of Medicine, Philadelphia, PA, USA

3 St Luke's University Health Network, 240 Cetronia Road, Suite 205 North, Allentown, PA 18104, USA
$(B M I) \geq 120 \%$ of the 95 th percentile for their age and sex. Comorbidities such as type two diabetes mellitus, hypertension, obstructive sleep apnea, and non-alcoholic fatty liver disease $[2,3]$ are associated with excess body weight.

Evidence of long-term weight control following lifestyle interventions in children is scarce. In addition, the majority of these interventions in adults proved ineffective in longterm weight control $[4,5]$. Bariatric surgery in adults is widely accepted as a treatment for obesity. Moreover, the effect of bariatric surgery has proven to persist over many years, leading to a reduction in mortality and obesity-associated comorbidities [6-8].

Adolescence is a critical period for maturation. Risk-/reward-related regulatory function and obesity during this period put youth at far greater risk for poor developmental outcomes [9]. Recent data indicated that children with obesity are likely to carry obesity to adulthood along with an increased 
morbidity and mortality risk $[10,11]$. Furthermore, the risk of depression, anxiety, and personality disorders has risen among this population [12].

Currently, adolescents either with a BMI $\geq 35 \mathrm{~kg} / \mathrm{m}^{2}$ and major comorbidities or with a $\mathrm{BMI} \geq 40 \mathrm{~kg} / \mathrm{m}^{2}$ and minor comorbidities are candidates for bariatric surgery [13].

The Metabolic and Bariatric Accreditation and Quality Improvement Program (MBSAQIP) database is the largest national bariatric specific database and includes data on patients less than 19 years old. The purpose of this study was to analyze the outcomes and safety of bariatric surgery in adolescents using the MBSAQIP database from 2015 to 2017 and to compare outcomes of bariatric surgery in adolescents to that of the adult population.

\section{Methods}

The MBSAQIP Participant User File (PUF) is the largest bariatric-specific clinical database and includes de-identified bariatric cases performed in MBSAQIP-accredited bariatric centers in the USA and Canada. The PUF contains data on preoperative patient demographics and comorbidities, intraoperative and postoperative variables, laboratory values, and 30day morbidity outcomes. The institutional review board (IRB) exempted our study from full board review due to its retrospective design.

According to the World Health Organization (WHO), an adolescent is any person between ages 10 and 19 . We used the 2015-2017 MBSAQIP PUF to identify all patients who are 19 years old or younger. We selected all primary laparoscopic sleeve gastrectomy (LSG) and laparoscopic Roux-en-Y gastric bypass (LRYGB) procedures. All revisions were excluded from our analysis.

Our primary outcomes included serious adverse events (SAEs), organ space infection (OSI), 30-day reoperation, and 30-day re-intervention rates. Secondary outcomes included operation length, length of stay, and readmission rates. OSI was defined as an infection that occurs within 30 days after the principal operative procedure and involves any of the anatomy (e.g., organs or spaces), other than the incision, which was opened or manipulated during the operation and at least one of the following:

A. Purulent drainage from a drain that is placed through a stab wound into the organ/space. This does not apply to drains placed during the principal operative procedure, which are continually in place, with continual evidence of drainage/infection since the time of the principal operative procedure.

B. Organisms isolated from an aseptically obtained culture of fluid or tissue in the organ/space.
C. An abscess or other evidence of infection involving the organ/space that is found on direct examination, during reoperation, by histopathologic or radiologic examination.

SAEs were defined as drain present at 30 days, unplanned intensive care unit (ICU) admission, intraoperative or postoperative cardiac arrest requiring cardiopulmonary resuscitation (CPR), intraoperative or postoperative stroke/cerebral vascular accident (CVA), coma $>24 \mathrm{~h}$, deep incisional surgical site infection (SSI), acute renal failure requiring dialysis, pulmonary embolism, intraoperative or postoperative myocardial infarction, postoperative vein thrombosis requiring therapy, pneumonia, ventilator $>48 \mathrm{~h}$, septic shock, or anticoagulation initiated for presumed/confirmed venous thrombosis or pulmonary embolus. The MBSAQIP definitions of all events included in our SAE composite score are available online at www.mbsaqip.org.

$p$ values were calculated based on separate Mann-Whitney rank sum tests, chi-square, or Fisher's exact tests as appropriate, with $p<0.05$ denoting statistical significance and no adjustment for the multiple comparisons. SPSS version 25 (Armonk, NY: IBM Corp) was used for data analysis.

\section{Results}

Table 1 shows demographics of adolescent patients $\leq 19$ years old. We found 1983 adolescent patients entered in MBSAQIP between 2015 and 2017 who underwent either a LRYGB or LSG. The average age and BMI were 18.1 and 47.5. Among the adolescents, 1568 (79.1\%) were females and 415 (20.9\%) were males. Among all adolescent patients, $78.2 \%$ underwent LSG and $21.8 \%$ underwent LRYGB.

Adolescents who underwent LSG had a significantly lower incidence of SAE compared with those who underwent a LRYGB (2.9\% vs $6.5 \%$, respectively; $p<0.05)$. The incidence of re-intervention within 30 days was also significantly lower following LSG (1.2\% vs 3\% for LSG and LRYGB, respectively; $p<0.05$ ). The incidence of reoperation was not significantly different, following LSG compared with LRYGB ( $1.1 \%$ vs $2.3 \% ; p=0.05)$ (Table 2$)$. In addition, the incidence of 30-day readmissions was lower for LSG compared with LRYGB $(2.6 \%$ vs $5.6 \% ; p<0.05)$. On average, LSG took significantly less time to perform compared with LRYGB (74 min vs 98 min for LSG and LRYGB, respectively; $p<0.05)$ (Table 3).

The average pre-operative BMI of adolescents who underwent surgery was higher than those of adults who underwent surgery (47.5 vs 45.2 for adolescents and adults, respectively). Compared to the adult population reported in MBSAQIP between 2015 and $2017(n=353,726)$, we did not find any significant difference in the incidence of our 
Table 1 Demographics

\begin{tabular}{llll}
\hline & Age $\left(\right.$ mean $\left.\pm \mathrm{SD}^{\mathrm{b}}\right)$ & Gender $(n, \%)$ & $\begin{array}{l}\text { Pre-operative BMI } \\
\left(\text { mean }+\mathrm{SD}^{\mathrm{b}}\right)\end{array}$ \\
\hline $\operatorname{LRYGB}^{\mathrm{a}}(n=431)$ & $18.2 \pm 1.0$ & 348 female $(80.7 \%)$ & $49.0 \pm 8.3$ \\
$\mathrm{LSG}^{\mathrm{a}}(n=1552)$ & $18.0 \pm 1.1$ & 83 male $(19.35)$ & $47.2 \pm 8.2$ \\
& $18.1 \pm 1.1$ & 332 male $(21.4 \%)$ & $47.5 \pm 8.3$ \\
Total $(N=1983)$ & & 1568 female $(79.1 \%)$ & \\
& & 415 male $(20.9 \%)$ & \\
\hline
\end{tabular}

${ }^{a}$ LRYGB: laparoscopic Roux-en-Y gastric bypass; LSG: laparoscopic sleeve gastrectomy

${ }^{\mathrm{b}} \mathrm{SD}$ : standard deviation

${ }^{\mathrm{c}}$ BMI: body mass index

primary and secondary outcomes except for the operation length which was longer in the adult population $(89.5 \mathrm{~min}$ vs $81.2 \mathrm{~min}$ for adults and adolescents, respectively; $p<0.05)$. Of note, readmission rates were not significantly different $(3.3 \%$ vs $4.1 \%$ for adolescents and adults, respectively; $p=0.06$ ) (Table 4).

\section{Discussion}

The twenty-first century has seen an enormous rise in the prevalence of obesity, reaching epidemic proportions among all age groups. Healthcare professionals have a critical role to play in preventing and managing obesity and its associated comorbidities [14]. The prevalence of the most severe class of obesity, class 3 obesity, defined as a BMI that is $\geq 140 \%$ higher than the 95th percentile (BMI of $\geq 40$ ), doubled in adolescents between 1999 and 2012 [15]. These rates imply that nearly 6 million children and adolescents in this country are carrying enormous fat reserves on their rapidly developing bodies during a critical period of their physical and psychological development [15].

Adolescents with severe obesity are at elevated risk of developing adult conditions, including type 2 diabetes, obstructive sleep apnea, high blood pressure, joint problems, and nonalcoholic fatty liver disease [16]. These conditions place adolescents at increased risk of developing chronic health problems early in life. Non-alcoholic fatty liver disease, for example, is now the leading cause of liver failure in adolescents [17].

In recent years, bariatric surgery in adolescents has received significant attention and has been the topic of numerous publications [18-20]. The safety and effectiveness of bariatric surgery in this population has been evaluated in multiple studies including a number of meta-analysis and systematic reviews [21-23]. The outcomes of these studies have led authors to conclude that bariatric surgery is an acceptable

Table 2 Primary outcomes

\begin{tabular}{|c|c|c|c|c|c|c|c|}
\hline & $\begin{array}{l}\text { SAEs }^{\mathrm{b}} \\
n(\%)\end{array}$ & $\mathrm{OSIs}^{\mathrm{c}}$ & $\begin{array}{l}\text { Operation length } \\
\text { (min) } \\
\text { (Median, range) }\end{array}$ & $\begin{array}{l}\text { Length of stay } \\
\text { (days) } \\
\text { (Median, range) }\end{array}$ & $\begin{array}{l}\text { At least one } \\
\text { reoperation } \\
\text { within } 30 \text { days } \\
(n, \%)\end{array}$ & $\begin{array}{l}\text { At least one } \\
\text { readmission } \\
\text { within } 30 \text { days } \\
(n, \%)\end{array}$ & $\begin{array}{l}\text { At least one } \\
\text { intervention } \\
\text { within } 30 \text { days } \\
(n, \%)\end{array}$ \\
\hline $\operatorname{LRYGB}^{\mathrm{a}}(n=431)$ & $28(6.5 \%)$ & $1(.2 \%)$ & $\begin{array}{l}98(37-606) \\
\text { Mean }=108.5\end{array}$ & $\begin{array}{l}2(0-17) \\
\text { Mean }=2.0\end{array}$ & $10(2.3 \%)$ & $24(5.6 \%)$ & $13(3 \%)$ \\
\hline $\mathrm{LSG}^{\mathrm{a}}(n=1552)$ & $45(2.9 \%)$ & $4(.3 \%)$ & $\begin{array}{l}66(1-395) \\
\text { Mean =73.8 }\end{array}$ & $\begin{array}{l}2(0-32) \\
\text { Mean }=1.7\end{array}$ & $17(1.1 \%)$ & $41(2.6 \%)$ & $18(1.2 \%)$ \\
\hline$p$ value $^{\mathrm{d}}$ & $<.0001$ & .93 & $<.0001$ & $<.0001$ & .05 & .003 & .006 \\
\hline Total $(N=1983)$ & $73(3.7 \%)$ & $5(.3 \%)$ & $\begin{array}{l}72(1-606) \\
\text { Mean }=81.2\end{array}$ & $\begin{array}{l}2(0-32) \\
\text { Mean }=1.7\end{array}$ & $27(1.4 \%)$ & $65(3.3 \%)$ & $31(1.6 \%)$ \\
\hline
\end{tabular}

${ }^{\text {a } L R Y G B: ~ l a p a r o s c o p i c ~ R o u x-e n-Y ~ g a s t r i c ~ b y p a s s ; ~ L S G: ~ l a p a r o s c o p i c ~ s l e e v e ~ g a s t r e c t o m y ~}$

${ }^{\mathrm{b}}$ SAEs: severe adverse events, including the following: drain present at 30 days; unplanned ICU admission, intraoperative or postoperative cardiac arrest requiring CPR, intraoperative or postoperative stroke/cerebral vascular accident (CVA), coma $>24 \mathrm{~h}$, deep incisional SSI, acute renal failure requiring dialysis, pulmonary embolism, intraoperative or postoperative myocardial infarction, postoperative vein thrombosis requiring therapy, pneumonia, ventilator $>48 \mathrm{~h}$, septic shock, anticoagulation initiated for presumed/confirmed venous thrombosis or pulmonary embolus

${ }^{\mathrm{c}}$ OSI: organ space infection

${ }^{\mathrm{d}}$ Based on separate Mann-Whitney rank sums tests, chi-square, or Fisher's exact tests as appropriate, with $p<.05$ denoting statistical significance and no adjustment for the multiple comparisons 
Table 3 Intervention rates

\begin{tabular}{lll}
\hline & Gender $(n, \%)$ & Pre-operative BMI $\left(\right.$ mean $\left.+\mathrm{SD}^{\mathrm{a}}\right)$ \\
\hline Adult $(>19$ years $)(n=353,726)$ & 281,908 female $(79.7 \%)$ & $45.2 \pm 8.1$ \\
& 71,818 male $(20.3 \%)$ & \\
Adolescent $(\leq 19$ years $)(n=1983)$ & 1568 female $(79.1 \%)$ & $47.5 \pm 8.3$ \\
& 415 male $(20.9 \%)$ & \\
Total $(N=355,709)$ & 283,476 female $(79.7 \%)$ & $46.4 \pm 8.2$ \\
& 72,233 male $(20.3 \%)$ & \\
\hline
\end{tabular}

${ }^{\text {a }} \mathrm{SD}$ : standard deviation

${ }^{\mathrm{b}}$ BMI: body mass index

treatment option for severe obesity in the adolescent population [18-23]. Some of these studies have focused on 30-day outcomes similar to our study $[24,25]$. In a study using data from the University HealthSystem Consortium, Varela et al. reported similar complication rates and mortality between adolescents and adults who underwent bariatric surgery [24]. Inge et al. further confirmed the safety profile of bariatric surgery in adolescents in a prospective, multisite observational study of 242 patients [25]. However, despite the recent increase in publications on this topic, to our knowledge, this is the first study to report on the outcomes of bariatric surgery in adolescents based on a national bariatric specific database (MBSAQIP) and to compare the outcomes of surgery in adolescents to that of adults. Additionally, this is based on a larger sample size of adolescents and uses more recent data than the majority of available literature.

Numerous outcomes were reported in this study in order to assess the safety of LSG and LRYGB. We have previously reported on the use of SAE and OSI as an overall measure of outcomes using MBSAQIP [26]. In addition to SAE and OSI, we also reported 30-day re-operations and re-interventions which can be a source of concern for any parents whose child is undergoing surgery.

In addition to the incidence of 30-day adverse events which are very important to clinicians and parents alike, we also looked at readmission rates because readmission rates are a major concern in accredited bariatric centers and arguably one of the most important outcome measures in bariatric surgery. Readmissions can lower reimbursement rates, affect patient satisfaction scores, and also result in increasing healthcare costs.

Our results showed that SAE, intervention, and readmission rates are lower following LSG compared with LRYGB. LSG was also the most commonly performed bariatric procedure in adolescents comprising $78.2 \%$ of procedures among patients who qualified for our study. We have previously reported on the outcomes of LSG and LRYGB in adults and found similar results [27]. LRYGB is typically more invasive

Table 4 Secondary outcomes

\begin{tabular}{|c|c|c|c|c|c|c|c|}
\hline & $\begin{array}{l}\mathrm{SAEs}^{\mathrm{a}} \\
n(\%)\end{array}$ & $\begin{array}{l}\text { OSIs }^{\mathrm{b}} \\
n(\%)\end{array}$ & $\begin{array}{l}\text { Operation } \\
\text { length (min) } \\
\text { (Median, range) }\end{array}$ & $\begin{array}{l}\text { Length of stay } \\
\text { (days) } \\
\text { (Median, range) }\end{array}$ & $\begin{array}{l}\text { At least one } \\
\text { reoperation } \\
\text { within } 30 \text { days } \\
(n, \%)\end{array}$ & $\begin{array}{l}\text { At least one } \\
\text { readmission } \\
\text { within } 30 \text { days } \\
(n, \%)\end{array}$ & $\begin{array}{l}\text { At least one } \\
\text { intervention } \\
\text { within } 30 \text { days } \\
(n, \%)\end{array}$ \\
\hline $\begin{array}{l}\text { Adult } \\
(>19 \text { years }) \\
(n=353,726)\end{array}$ & $14,475(4.1 \%)$ & $\begin{array}{l}846 \\
(.2 \%)\end{array}$ & $\begin{array}{l}77(1-720) \\
\text { Mean }=89.5\end{array}$ & $\begin{array}{l}2(0-139) \\
\text { Mean }=1.8\end{array}$ & $4761(1.3 \%)$ & $14,509(4.1 \%)$ & $5208(1.5 \%)$ \\
\hline $\begin{array}{l}\text { Adolescent } \\
\quad(\leq 19 \text { years }) \\
(n=1983)\end{array}$ & $73(3.7 \%)$ & $5(.3 \%)$ & $\begin{array}{l}72(1-606) \\
\text { Mean }=81.2\end{array}$ & $\begin{array}{l}2(0-32) \\
\text { Mean }=1.7\end{array}$ & $27(1.4 \%)$ & $65(3.3 \%)$ & $31(1.6 \%)$ \\
\hline$p$ value $^{\mathrm{c}}$ & .35 & .91 & $<.0001$ & .87 & .96 & .06 & .74 \\
\hline Total $(N=355,709)$ & $\begin{array}{l}14,548 \\
(4.1 \%)\end{array}$ & & $\begin{array}{l}77(1-720) \\
\text { Mean }=89.4\end{array}$ & $\begin{array}{l}2(0-139) \\
\text { Mean }=1.8\end{array}$ & $4788(1.3 \%)$ & $14,574(4.1 \%)$ & $5239(1.5 \%)$ \\
\hline
\end{tabular}

${ }^{\text {a }}$ SAEs: severe adverse events, including the following: drain present at 30 days, unplanned ICU admission, intraoperative or postoperative cardiac arrest requiring CPR, intraoperative or postoperative stroke/cerebral vascular accident (CVA), coma $>24 \mathrm{~h}$, deep incisional SSI, acute renal failure requiring dialysis, pulmonary embolism, intraoperative or postoperative myocardial infarction, postoperative vein thrombosis requiring therapy, pneumonia, ventilator $>48 \mathrm{~h}$, septic shock, anticoagulation initiated for presumed/confirmed venous thrombosis or pulmonary embolus

${ }^{\mathrm{b}}$ OSI: organ space infection

${ }^{\mathrm{c}}$ Based on separate Mann-Whitney rank sums tests, chi-square, or Fisher's exact tests as appropriate, with $p<.05$ denoting statistical significance and no adjustment for the multiple comparisons 
and can result in a higher rate of complications. However, the decision to undergo either LSG or LRYGB should be individualized because other factors need to be taken into account like excess weight loss and long-term success. Although there is limited long-term data available regarding outcomes after bariatric surgery in adolescents, studies in adult populations have suggested that RYGB may result in greater \%EWL, improved dyslipidemia outcomes, and a lower incidence of postoperative gastro-esophageal reflux disease [28]. All this data should be discussed with families in the context of a multidisciplinary approach before a surgical procedure is chosen.

Outcomes of bariatric surgery in adolescents are of crucial importance to clinicians and parents. Any complication in an adolescent can have substantial implications on the adolescent's future as well as his or her entire family. Although the safety and efficacy of bariatric surgery have been well established [18-23], there are limited studies comparing the safety of bariatric surgery in adolescents to that of adults based on larger sample sizes. In our study, we found no difference in outcomes between adults and adolescents which suggest that in the context of a comprehensive and multidisciplinary program, adolescents can safely undergo bariatric surgery and expect outcomes similar to that of bariatric surgery in adults. Of note, the readmission rate in adolescents was no different than that of adults. There was a trend for lower readmission rate in adolescents. This may be attributable to better preoperative education and closer postoperative follow-up and better support system at home given the age of the patient.

The study has several limitations. First, the definition of SAE in our manuscript only includes complications that result in intervention or reoperation; other complications, which may be considered significant by patients and providers, were not included. For example, patients who develop pulmonary embolism (PE) or a deep venous thrombosis (DVT) and managed by anticoagulation, although considered significant by many, were not included in our definition of SAE. Second, our model does not capture any patient who develops a SAE after the first 30 days because the PUF was limited to the first 30 days. Patients who develop a late leak following LSG, for example, are not captured by our model. Additionally, bleeding is not directly reported in the MBSAQIP PUF unless the patient received a transfusion. Our study captures transfusions as an intervention within 30 days of operation but does not directly evaluate bleeding as an outcome. We also did not include any band patients. Although LSG and LRYGB are the two most commonly performed procedures, certain pediatric obesity management centers are still performing band placement. Additionally, the data is based on the analysis of a clinical database entered in a prospective fashion with all the inherent limitations of such a database including the inability to account for some confounding factors such as operative technique, institution specific protocols, and the indications for reoperation and re-intervention Also, the data does not include any outcome measures from beyond the first 30 days following surgery. Although most serious complications occur within the first 30 days, long-term complications following bariatric surgery can always occur and are of significant consequence in adolescents. These long-term outcomes are unfortunately not captured in our study. Lastly, it is difficult to determine the clinical meaningfulness of statistically significant differences in outcomes. Given that adjustments were not made for the multiple comparisons, there is a possibility that type I errors could account for these differences.

\section{Conclusion}

Bariatric surgery in adolescents appears to have a safety profile that is similar to that of adults based on analysis of the MBSAQIP database. In adolescents, patients who underwent LSG had fewer SAE, re-intervention, and readmission rates compared with patients who underwent LRYGB. There was no difference in 30-day outcomes between adolescents and adults.

\section{Compliance with Ethical Standards}

The institutional review board (IRB) exempted our study from full board review due to its retrospective design.

Conflict of Interest The authors declare that they have no conflict of interest.

Ethical Approval Statement For this type of study, formal consent is not required.

Informed Consent Statement Does not apply.

\section{References}

1. Casey BJ, Jones R, Hare T. The adolescent brain. Ann N Y Acad Sci. 2008;1124:111-26.

2. ElChaar M, Lundberg P, Stoltzfus J. Thirty-day outcomes of sleeve gastrectomy versus Roux-en-Y gastric bypass: first report based on metabolic and bariatric surgery accreditation and quality improvement program database. Surg Obes Relat Dis. 2019;15(1):1-7.

3. Colquitt JL, Pickett K, Loveman E, et al. Surgery for weight loss in adults. Cochrane Database Syst Rev. 2014;8:CD003641.

4. Daniels SR. Complications of obesity in children and adolescents. Int J Obes. 2009;33:S60-5.

5. Durkin N, Desai A. What is the evidence for paediatric/adolescent bariatric surgery? Curr Obes Rep. 2017;6(3):278-85.

6. Ebbeling CB, Pawlak DB, Ludwig DS. Childhood obesity: publichealth crisis, common sense cure. Lancet. 2002;360(9331):473-82.

7. Fouse T, Brethauer S. Resolution of comorbidities and impact on longevity following bariatric and metabolic surgery. Surg Clin North Am. 2016;96(4):717-32.

8. Gill RS, Majumdar SR, Rueda-Clausen CF, et al. Comparative effectiveness and safety of gastric bypass, sleeve gastrectomy and 
adjustable gastric banding in a population-based bariatric program: prospective cohort study. Can J Surg. 2016;59(4):233-41.

9. Han JC, Lawlor DA, Kimm SYS. Childhood obesity. Progress and challenges. Lancet. 2010;375(9727):1737-48.

10. Harris KM, Perreira K, Lee D. Obesity in the transition to adulthood: predictions across race/ethnicity, immigrant generation, and sex. Arch Pediatr Adolesc Med. 2009;163(11):1022-8.

11. Hsia DS, Fallon SC, Brandt ML. Adolescent bariatric surgery. Arch Pediatr Adolesc Med. 2012;166(8):757-66.

12. Rankin J, Matthews L, Cobley S, et al. Psychological consequences of childhood obesity: psychiatric comorbidity and prevention. Adolesc Health Med Ther. 2016;7:125-46.

13. Michalsky $\mathrm{M}$, Reichard $\mathrm{K}$, Inge $\mathrm{T}$, et al. ASMBS Pediatric Committee best practice guidelines. Surg Obes Relat Dis. 2012;8: $1-7$.

14. Reilly JJ, Kelly J. Long-term impact of overweight and obesity in childhood and adolescence on morbidity and premature mortality in adulthood: systematic review. Int J Obes. 2011;35(7):891-8.

15. Skinner AC, Skelton JA. Prevalence and trends in obesity and severe obesity among children in the United States, 1999-2012. JAMA Pediatr. 2014;168(6):561-6.

16. Norris SL, Kansagara D, Bougatsos C, et al. Screening adults for type 2 diabetes: a review of the evidence for the U.S. Preventive Services Task Force. Ann Intern Med. 2008;148(11):855-68.

17. Welsh JA, Karpen S, Vos MB. Increasing prevalence of nonalcoholic fatty liver disease among United States adolescents, 19881994 to 2007-2010. J Pediatr. 2013;162(3):496-500.e1.

18. Inge TH, Courcoulas AP, Jenkins TM, et al. Weight loss and health status 3 years after bariatric surgery in adolescents. N Engl J Med. 2016;374(2):113-23.

19. Inge TH, Jenkins TM, Xanthakos SA, et al. Long-term outcomes of bariatric surgery in adolescents with severe obesity (FABS-5+): a prospective follow-up analysis. Lancet Diabetes Endocrinol. 2017;5(3): 165-73.

20. Olbers T, Beamish AJ, Gronowitz E, et al. Laparoscopic roux-en-Y gastric bypass in adolescents with severe obesity (AMOS): a prospective, 5-year, Swedish nationwide study. Lancet Diabetes Endocrinol. 2017;5:174-83.

21. Pedroso FE, Angriman F, Endo A, et al. Weight loss after bariatric surgery in obese adolescents: a systematic review and meta-analysis. Surg Obes Relat Dis. 2018;14(3):413-22.

22. Shoar S, Mahmoudzadeh H, Naderan M, et al. Long-term outcome of bariatric surgery in morbidly obese adolescents: a systematic review and meta-analysis of 950 patients with a minimum of 3 years follow-up. Obes Surg. 2017;27(12):3110-7.

23. Childerhose JE, Alsamawi A, Mehta T, et al. Adolescent bariatric surgery: a systematic review of recommendation documents. Surg Obes Relat Dis. 2017;13(10):1768-79.

24. Varela JE, Hinojosa MW, Nguyen NT. Perioperative outcomes of bariatric surgery in adolescents compared with adults at academic medical centers. Surg Obes Relat Dis. 2007;3(5):537-40.

25. Inge $\mathrm{TH}$, Zeller $\mathrm{MH}$, Jenkins $\mathrm{TM}$, et al. Perioperative outcomes of adolescents undergoing bariatric surgery: the Teen-Longitudinal Assessment of Bariatric Surgery (Teen-LABS) study. JAMA Pediatr. 2014;168(1):47-53.

26. El Chaar M, Stoltzfus J, Gersin K, et al. A novel risk prediction model for 30-day severe adverse events and readmissions following bariatric surgery based on the MBSAQIP database. Surg Obes Relat Dis. 2019;15(7):1138-45.

27. El Chaar M, Hammoud N, Ezeji G, et al. Laparoscopic sleeve gastrectomy versus laparoscopic Roux-en-Y gastric bypass: a single center experience with 2 years follow-up. Obes Surg. 2015;25(2):254-62.

28. Sharples AJ, Mahawar K. Systematic review and meta-analysis of randomised controlled trials comparing long-term outcomes of Roux-en-Y gastric bypass and sleeve gastrectomy. Obes Surg. 2020;30(2):664-72.

Publisher's Note Springer Nature remains neutral with regard to jurisdictional claims in published maps and institutional affiliations. 